\title{
Reversible cerebral vasoconstriction syndrome
}

\author{
Gooneratne I K ${ }^{1,2}$, Dassanayake $\mathbf{S}^{2}$, Ganewatta $\mathbf{E}^{2}$ \\ Journal of the Ceylon College of Physicians, 2019, 50, 87-89
}

\begin{abstract}
Reversible cerebral vasoconstriction syndrome (RCVS) is an increasingly recognized cause of acute headache. Postpartum reversible vasoconstriction syndrome is a recognized subset of RCVS. Differentiating RCVS from primary angiitis of the central nervous system can be dificult but has important therapeutic implications. This case highlights the clinical and imaging features of RCVS and stresses the importance of diagnosing this rare entity.
\end{abstract}

Key words: reversible cerebral vasoconstriction syndrome, postpartum reversible vasoconstriction syndrome, postpartum headache

\section{Introduction}

RCVS is a lesser known clinical entity characterized by thunderclap headaches and reversible constriction of cerebral arteries. Headache mimics subarachnoid haemorrhage $(\mathrm{SAH})$ and the diffuse and segmental constriction of cerebral arteries resolves within 3 months $^{1}$. The pathophysiology is unknown. Females are most affected and over $50 \%$ of cases occur in the puerperium or after exposure to vasoactive substances ${ }^{2}$.

\section{Case report}

A 31-year-old woman was admitted to a tertiary care hospital with three consecutive episodes of spontaneous severe thunderclap headaches arising from the back of the neck with nausea on the $7^{\text {th }}$ day of post-partum. The maximum intensity of the pain was within one minute of onset and headaches lasted for less than 2 hours each time. She was prescribed citalopram $20 \mathrm{mg}$ in the past for depressive symptoms and anxiety which she discontinued since conception. Her neurological examination was normal.

Her full blood count and biochemical analysis were normal. Brain computed tomography with contrast and MRI scans were unremarkable. Magnetic resonance (MR) angiography demonstrated multiple stenotic segments in both anterior and posterior circulations (Figure 1). MR venography was normal. Probabilities of $\mathrm{SAH}$ and cerebral venous thrombosis were excluded. Digital subtraction angiography (DSA) showed multiple segments of narrowing in vessel caliber. Laboratory examinations including lipid profile, prothrombin time, activated partial thromboplastin time, levels of protein $\mathrm{C}$, protein $\mathrm{S}$, anti-thrombin III, antiphospholipid antibodies, complement, anti-neutrophil cytoplasm antibody, anti-nuclear antibody and antidouble-stranded DNA antibody, anti-Ro/SSA and antiLa/SSB, ASO, homocysteine, anti-HBs, HBsAg, antiHCV, anti-HIV, VDRL were unremarkable. CSF analysis was within normal limits (cells - nil, protein$39 \mathrm{mg} / \mathrm{dl}$, glucose $-79 \mathrm{mg} / \mathrm{dl}$ ).

Based on the imaging studies, the two probable diagnoses entertained were RCVS and primary angiitis of the central nervous system (PACNS). Based on the clinical history, postpartum RCVS was suspected and she was treated with calcium channel blocker, nimodipine $360 \mathrm{mg}$ daily and acetylsalicylic acid 300 mg daily. She remained asymptomatic and follow-up MR angiography at 12 weeks demonstrated normal vasculature. Thus, a definitive diagnosis of RCVS was established.

\footnotetext{
${ }^{1}$ National Hospital of Sri Lanka, ${ }^{2}$ Colombo North Teaching Hospital, Sri Lanka.

Corresponding author: GIK, e-mail: kishig@gmail.com
}

This is an open-access article distributed under the terms of the Creative Commons Attribution License, which permits unrestricted use, distribution, and reproduction in any medium, provided the original author and source are credited. 


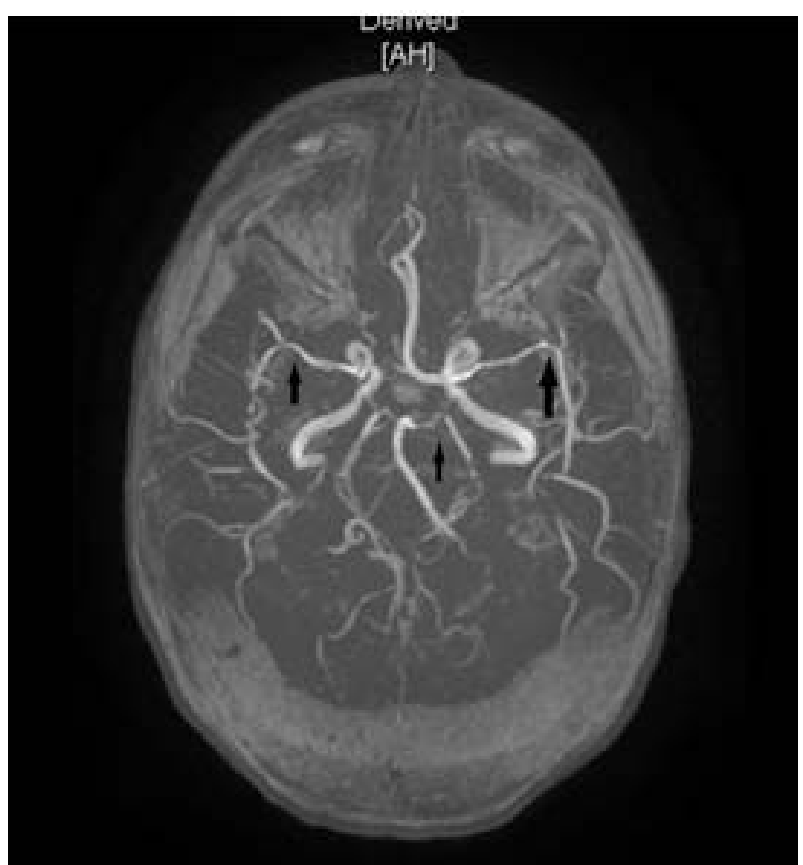

Figure 1. MRA - Demonstrates multiple constrictions (arrows) suggestive of segmental stenosis of both anterior and posterior circulations.

\section{Discussion}

RCVS is a rare and a serious cause of headache and postpartum RCVS occurs in the early postpartum period $^{2}$. The diagnosis of the index case was based on the following diagnostic criteria (our case met all criteria) ${ }^{3}$ :

(1) Severe, acute headaches, with or without additional neurological signs or symptoms.

(2) Angiography documenting multifocal segmental cerebral artery vasoconstriction.

(3) No evidence of aneurysmal subarachnoid hemorrhage.

(4) Normal or near-normal cerebrospinal fluid analysis.

(5) Reversibility of angiographic abnormalities within 12 weeks of symptom onset.

A common symptom of RCVS is an acute severe thunderclap headache which is defined as a severe headache reaching maximal intensity within one minute. The headache tends to be recurrent, over a period of days to weeks ${ }^{4}$. Our patient however presented on the day one of the illness. The typical headache is bilateral, although it may be unilateral, starting in the occipital region, followed by diffuse pain.
It may occur spontaneously or be precipitated by exercise or a Valsalva manoeuvre. The headache can often be accompanied by nausea, vomiting, photophobia and phonophobia ${ }^{1}$. In a patient with acute severe headache, a number of other intracranial disorders such as aneurysmal subarachnoid haemorrhage, cerebral venous sinus thrombosis, intracranial haemorrhage and pituitary apoplexy should be considered in addition to RCVS. When recurrent thunderclap headaches are experienced within 1-2 weeks by the patient, RCVS is the most likely diagnosis.

RCVS, cervical artery dissection, subarachnoid haemorrhage and cerebral venous thrombosis were considered in the initial differential diagnosis. However, imaging was characteristic for RCVS in our patient which was segmental vasoconstriction of the cerebral arteries demonstrated both on DSA and MRA. The segmental vasoconstriction was reversible within a 12week period $^{1}$, in our patient. Angiographic changes in cerebral arteries, described as a "string of beads", are highly characteristic of $\mathrm{RCVS}^{4}$ while CT and MR imaging is often normal ${ }^{1}$.

PACNS is radiologically identical to RCVS, making the clinical history, risk factors and CSF studies important in differentiating these two conditions ${ }^{2}$. Distinction is important, though dificult, because the treatment is quite different since PACNS requires immunotherapy including glucocorticoid therapy. The reversibility of the angiographic features is the key to the diagnosis of RCVS but only helpful retrospectively. Clinical features of PACNS at diagnosis are highly variable and nonspecific; classic or pathognomonic clinical symptoms are lacking. Major symptoms of cerebral vasculitis are headache (60\%), altered cognition (50\%) and focal neurologic deficits (e.g. hemiparesis, ataxia, aphasia, dysarthria and visual disturbances) ${ }^{4}$. The headache of PACNS has an insidious onset, progressing gradually, and thus distinct from that typical of RCVS. Thus, if a patient presents with a classic history of repetitive thunderclap headaches, had no evidence of SAH, had normal CSF analysis and a normal MRI, and showed findings to suggest RCVS on vascular imaging; a diagnosis of RCVS can be made.

Vasodilators, including calcium channel blockers are often used to treat RCVS based on observational studies and anecdotal evidence ${ }^{5}$. However, calcium channel blockers with systemic action which reduces systolic blood pressure, can compromise the cerebral perfusion in patients with severe vasoconstriction, and should therefore be used with caution. Nimodipine is 
the most used and may have a more selective action on cerebral circulation ${ }^{2}$. Despite reducing the number of episodes and the intensity of the headache, prospective and retrospective studies suggest that it does not affect the time course of cerebral vasoconstriction. It may be given orally or intravenously at a dose used in the treatment of SAH for a duration between 4 to 12 weeks. Glucocorticoids were previously considered a potential treatment; however, recently steroids have been shown to be an independent predictor of poor outcome and should be avoided 6 .

RCVS can be complicated by ischemic infarcts, convexity SAH, lobar intracerebral haemorrhage, and cerebral oedema ${ }^{5}$. Even with these complications the rate of permanent neurological disability is surprisingly low.

\section{Conclusion}

There are characteristic features in the history and on neuroimaging that are distinct for RCVS. However, overlapping features with other conditions can make the diagnosis difficult. Distinguishing RCVS from PACNS is important, as glucocorticoid treatment indicated for PACNS can be harmful in RCVS. The management of RCVS is predominantly supportive.

\section{References}

1. Santos L, Azevedo E. Reversible cerebral vasoconstriction syndrome; Anarrative revision of the literature. Porto Biomed J. $2016 ; 1$ (2): $65-71$.

2. Skeik N, Porten BR, Kadkhodayan Y, McDonald W, Lahham F. Postpartum reversible cerebral vasoconstriction syndrome: review and analysis of the current data. Vasc Med. 2015; 20(3): 256-65.

3. Calabrese LH, Dodick DW, Schwedt TJ, Singhal AB. Narrative Review: Reversible Cerebral Vasoconstriction Syndromes. Ann Intern Med. 2007; 146: 34-44.

4. Berlit $\mathrm{P}, \mathrm{Kraemer} \mathrm{M}$. Cerebral vasculitis in adults: what are the steps in order to establish the diagnosis? Red flags and pitfalls. Clin Exp Immunol 2014; 175: 419-24.

5. Tan LH, Flower O. Reversible cerebral vasoconstriction syndrome: an important cause of acute severe headache. Emerg Med Int. 2012; 2012: 303152.

6. Singhal AB, Topcuoglu MA. Glucocorticoid-associated worsening in reversible cerebral vasoconstriction syndrome. Neurology 2017; 17: 88(3): 228-36. 